\title{
Infecções de repetição: o que é importante para o pediatra
}

\author{
Recurrent infections: what is important to pediatricians
}

\section{Pérsio Roxo Júnior ${ }^{1}$, Beatriz Tavares C. Carvalho², Fabíola Scancetti Tavares ${ }^{3}$}

\section{RESUMO}

Objetivo: Apresentar uma revisão atualizada sobre infecções de repetição em crianças, abordando importantes aspectos para o pediatra relacionados a infecções em crianças saudáveis e em crianças com imunodeficiências primárias.

Fontes de dados: Artigos relacionados ao tema foram coletados dos bancos de dados Medline e Lilacs no período entre 1980 e 2008, tendo sido selecionados artigos de meta-análise, revisão e estudos clínicos realizados em seres humanos, cuja metodologia e discussão estavam bem estruturadas. Também foram incluídos livros-texto nacionais e internacionais pertinentes ao tema.

Síntese dos dados: Infecções de repetição são frequentes na clínica pediátrica. Aproximadamente $50 \%$ dessas crianças são saudáveis e $10 \%$ podem ser imunodeficientes. A criança saudável apresenta crescimento e desenvolvimento normais e se encontra bem entre os episódios infecciosos. As infecções, na maioria das vezes, não têm curso prolongado ou complicado e ocorrem devido ao aumento da exposição a agentes infecciosos do meio ambiente nos primeiros anos de vida. As imunodeficiências primárias geralmente se manifestam como infecções de repetição por microorganismos específicos ou por germes de baixa virulência. $\mathrm{Na}$ maioria das vezes, os quadros infecciosos apresentam evolução prolongada, resposta inadequada à antibioticoterapia e elevados riscos de complicações.

Conclusões: O diagnóstico precoce das imunodeficiências primárias é essencial para que medidas terapêuticas sejam rapidamente instituídas, reduzindo os riscos de ocorrência de óbito e complicações.

Palavras-chave: infecções de repetição; imunidade; imunodeficiências primárias.

\section{ABSTRACT}

Objective: To present an up-to-date review about recurrent infections in children, addressing important aspects for pediatricians related to infections in healthy children and in children with primary immunodeficiencies.

Data source: Articles related to the subject were collected from Medline and Lilacs databases between 1980 and 2008, selecting articles of meta-analysis, review and clinical trials in humans, with well-structured methodology and discussion. National and international textbooks relevant to the subject were also included.

Data synthesis: Recurrent infections are frequent in pediatric clinics. Approximately $50 \%$ of these children are healthy and $10 \%$ may be immunodeficient. The healthy child presents normal growth and development and is well between infections. Most times, infections do not have prolonged or complicated evolution, and they occur due to exposure to infectious agents from the environment during the first years of life. Primary immunodeficiencies usually manifest as recurrent infections by specific microorganisms or by low virulence germs. Most of the times, these infections are prolonged, they present inadequate response to antibiotics and a high risk of complications.

Conclusions: An early diagnosis of primary immunodeficiencies is essential so that therapeutic measures may be taken quickly, reducing risks of death and complications.

Key-words: recurrent infections; immunity; primary immunodeficiencies.
Instituição: Departamento de Puericultura e Pediatria da Faculdade de Medicina de Ribeirão Preto da Universidade de São Paulo (FMRP-USP), Ribeirão Preto, SP, Brasil

1Professor assistente do Departamento de Puericultura e Pediatria da FMRP-USP, Ribeirão Preto, SP, Brasil

2Professora adjunto da Disciplina de Alergia, Imunologia Clínica e Reumatologia do Departamento de Pediatria da Universidade Federal de São Paulo (Unifesp), São Paulo, SP, Brasil

${ }^{3}$ Médica do Hospital Universitário da Universidade de Brasília (UnB), Brasília, DF, Brasil
Endereço para correspondência:

Pérsio Roxo Júnior

Avenida Bandeirantes, 3900

CEP 14049-900 - Ribeirão Preto/SP

persiorj@fmrp.usp.br

Recebido em: 26/9/08

Aprovado em: 19/12/08 


\section{Introdução}

Infecções respiratórias de repetição (IRR) são muito frequentes na prática clínica pediátrica e acometem número significativo de crianças com menos de 6 anos de idade. Os critérios mais utilizados para definir IRR são: ausência de quaisquer doenças de base que justifiquem as infecções de repetição (imunodeficiência primária ou secundária, fibrose cística, malformações das vias aéreas, síndrome dos cílios imóveis, dentre outras) e presença de, no mínimo, uma das seguintes condições: 1) seis ou mais infecções respiratórias por ano; 2) uma ou mais infecções respiratórias mensais; 3) três ou mais infecções anuais do trato respiratório inferior ${ }^{(1)}$.

É importante que essas crianças sejam submetidas a uma abordagem clínica diagnóstica, procurando identificar os fatores de risco que as tornam suscetíveis à repetição de processos infecciosos. De acordo com Conley e Stiehm ${ }^{(2)}$, aproximadamente metade das crianças com IRR é saudável, $30 \%$ têm alergia, $10 \%$ possuem patologias crônicas e $10 \%$ podem ter imunodeficiência.

Dessa forma, algumas recomendações são importantes para o pediatra:

1. Identificar se a criança apresenta alguma doença de base.

A presença de infecções com características similares, como pouca gravidade e curta duração, é bem diferente de infecções graves com curso prolongado e complicações, às vezes causadas por agentes oportunistas, acompanhadas de crescimento inadequado e, não raro, uma história familiar com morte precoce na família. Dessa forma, uma história clínica e exame físico detalhados são, na maioria das vezes, suficientes para excluir doenças mais graves ${ }^{(3,4)}$.

2. Atentar para fatores de risco relacionados às IRR em crianças (Quadro 1) ${ }^{(5)}$ e tentar removê-los. A influência das condições socioeconômicas e ambientais é muito importante. Há evidências de que a obesidade e a presença de infecções respiratórias prévias sejam fatores de risco relevantes para o desenvolvimento de IRR em adultos jovens ${ }^{(6)}$.

3. Reconhecer a existência de imaturidade do sistema imunológico adaptativo nos primeiros anos de vida, que predispõe a infecções de repetição, geralmente sem repercussão importante e sem que a criança tenha necessariamente alguma doença de base. O número dessas infecções se reduz com a idade ${ }^{(7)}$.
4. Identificar alergia. Os sintomas de rinite alérgica frequentemente são confundidos com os de infecções das vias aéreas superiores e a criança é equivocadamente diagnosticada como portadora de infecções respiratórias, não sendo submetida ao tratamento adequado para alergia. Crises de asma são muitas vezes equivocadamente diagnosticadas como broncopneumonias de repetição. No entanto, rinite alérgica e asma são importantes fatores de risco para o desenvolvimento de IRR tanto em crianças como em adultos jovens ${ }^{(6)}$. O diagnóstico dessas condições pode ser realizado por testes cutâneos de hipersensibilidade imediata para alérgenos intra e extradomiciliares, determinação sérica da IgE total e específica para alérgenos e espirometria. Essa propedêutica tem a finalidade, também, de orientar a adequada profilaxia ambiental, com o objetivo de reduzir a exposição aos alérgenos e otimizar o tratamento ${ }^{(8)}$.

5. Reconhecer o refluxo gastroesofágico, que frequentemente causa tosse noturna, sendo, dessa forma, muitas vezes confundido com infecções de vias aéreas. É comum a presença de manifestações extraesofágicas, como erosão dentária, sinusite, otite média recorrente, faringite, laringite, pneumonia e fibrose pulmonar idiopática, embora as mesmas sejam mais prevalentes em adultos do que em crianças. Dessa forma, são necessários métodos diagnósticos complementares dessa importante entidade, como monitorização do $\mathrm{pH}$ esofágico, endoscopia ou cintilografia ${ }^{(9)}$.

\section{Infecções na criança saudável}

Caracteristicamente, a criança saudável apresenta crescimento e desenvolvimento normais e encontra-se bem entre os episódios infecciosos. As infecções, na maioria das vezes, não têm curso prolongado ou complicado e ocorrem devido ao aumento da exposição a agentes infecciosos do meio ambiente nos primeiros anos de vida, como a frequência à creche, berçário ou escola, especialmente durante o outono

Quadro 1 - Fatores de risco para aumento de infecções respiratórias em crianças saudáveis, adaptado de Towns e Wong ${ }^{(5)}$
Frequentar creche ou escolinha Irmãos mais velhos
Exposição à fumaça de cigarro Poluição atmosférica
Casa com muitas pessoas
Baixo nível socioeconômico. 
e inverno ${ }^{(10,11)}$. Foi demonstrado que o risco de otite média de repetição é relacionado à idade de entrada na creche, com tendência à redução desse risco conforme a criança ingressamais tardiamente ${ }^{(12)}$. Outros fatores de risco para a ocorrência de otite média de repetição na criança saudável são desmame precoce, aglomeração, utilização de mamadeiras e chupetas, tabagismo domiciliar e infecção pelo vírus influenza ${ }^{(13,14,15)}$.

Outra importante questão a ser considerada é a imaturidade do sistema imunológico nos primeiros anos de vida. $\mathrm{O}$ recém-nascido apresenta redução progressiva dos níveis séricos de $\operatorname{IgG}$, adquiridos passivamente através de passagem transplacentária. Essa redução se dá por catabolismo e se inicia por volta do quarto mês de vida, estendendo-se até o oitavo mês na maioria dos lactentes. Esse período, que coincide com uma baixa capacidade de síntese própria de imunoglobulinas pelo lactente, é denominado hipogamaglobulinemia fisiológica ${ }^{(3)}$, no qual a criança pode apresentar IRR, na maioria das vezes sem repercussão clínica importante. Eventualmente, essa hipogamaglobulinemia pode se estender até 4 ou 5 anos de vida, normalizando-se após essa idade ${ }^{(16)}$.

Ainda no contexto da maturação imunológica, sabe-se que o lactente apresenta níveis bastante reduzidos de $\operatorname{Ig} \mathrm{A}$, que é a principal imunoglobulina presente nas mucosas dos tratos respiratório e gastrintestinal ${ }^{(17,18)}$. Dessa forma, níveis reduzidos de IgA em mucosas podem facilitar a penetração de alérgenos e patógenos através desses epitélios. Sob esse aspecto, o estímulo ao aleitamento natural é de suma importância, na medida em que o colostro e o leite materno fornecem grandes quantidades de IgA específica contra alguns patógenos respiratórios como Haemophilus influenzae, Streptococcus pneumoniae e vírus sincicial respiratório, especulando-se que esses anticorpos espalhados pela mucosa do trato respiratório possam impedir a aderência e a proliferação de tais patógenos, tanto em vias aéreas superiores como inferiores ${ }^{(19,20)}$.

Sabe-se, ainda, que lactentes têm capacidade limitada de produzir anticorpos específicos contra patógenos dotados de cápsula polissacarídica, como pneumococos, neissérias e Haemophilus influenzae B, o que os torna suscetíveis a infecções causadas por estes microorganismos nessa fase da vida ${ }^{(3)}$. A infecção pneumocócica permanece a maior causa de otite média, pneumonia, meningite e septicemia em várias partes do mundo, inclusive países desenvolvidos ${ }^{(21,22)}$. A vacina pneumocócica polissacarídica tem se mostrado ineficaz em crianças com menos de 2 anos de vida, por induzir uma resposta imunológica independente de células $\mathrm{T}^{(23)}$. Já a vacina pneumocócica conjugada é bastante eficaz na redução do número de infecções causadas pelos pneumococos nos primeiros
2 anos de vida, especialmente por sua capacidade de estimular uma resposta imunológica dependente de células $\mathrm{T}^{(24)}$.

\section{Infecções na criança com imunodeficiência primária}

As imunodeficiências primárias (IDP) ocorrem em 1:2.000 nascidos vivos, devendo ser encaradas como qualquer outra doença diagnosticada na rotina ambulatorial. Tratam-se de distúrbios de um ou mais componentes do sistema imunológico, sendo a quase totalidade dos casos de caráter congênito e hereditário ${ }^{(25)}$.

O grande marco no reconhecimento das IDP ocorreu em 1952, quando Ogden Bruton descreveu o primeiro paciente com redução da fração gamaglobulina na eletroforese de proteínas e infecções de repetição a se beneficiar da reposição de imunoglobulinas (agamaglobulinemia ligada ao $\mathrm{X})^{(26)}$. Desde então, mais que 150 IDP já foram descritas, contribuindo muito para o conhecimento do sistema imunológico normal pelos médicos.

A classificação das IDP vem se tornando complexa, de acordo com o setor da resposta imunológica comprometido (deficiências humorais, deficiências celulares, deficiências combinadas, deficiências fagocitárias e deficiências do complemento). As imunodeficiências humorais são as mais frequentes, correspondendo a aproximadamente $50 \%$ de todos os casos de IDP ${ }^{(27)}$.

O espectro clínico das IDP é amplo, geralmente se manifestando como infecções de repetição por microorganismos específicos ou por germes de baixa virulência. Embora as infecções possam ser de pequena gravidade, na maioria das vezes os quadros infecciosos apresentam evolução prolongada, resposta inadequada à antibioticoterapia habitualmente utilizada e elevados riscos de complicações e hospitalizações ${ }^{(25)}$. Pacientes com IDP podem apresentar também reações adversas graves após administração de vacinas constituídas de patógenos vivos, como a BCGite naqueles com doença granulomatosa crônica ou o desenvolvimento de poliomielite vacinal em pacientes com agamaglobulinemia ${ }^{(28)}$.

O tipo de patógeno e a localização das infecções podem sugerir a natureza do distúrbio imunológico (Tabela 1$)^{(29)}$. Imunodeficiências humorais cursam com infecções sinopulmonares por bactérias extracelulares encapsuladas e gastrintestinais por enterovírus e Giardia lamblia. Nas formas graves, podem ocorrer complicações como bronquiectasias, doenças gastrintestinais, malignidade e autoimunidade ${ }^{(29,30)}$. Deficiências da imunidade celular ocasionam infecções graves por patógenos de replicação intracelular, como vírus, fungos, 
Tabela 1 - Características das imunodeficiências primárias, adaptado de Woroniecka e Ballow ${ }^{(29)}$

\begin{tabular}{|c|c|c|c|c|}
\hline Características & $\begin{array}{l}\text { Defeito predominante } \\
\text { de célula T }\end{array}$ & $\begin{array}{l}\text { Defeito predominante } \\
\text { de célula B }\end{array}$ & Defeito de fagócito & $\begin{array}{l}\text { Defeito de } \\
\text { complemento }\end{array}$ \\
\hline Idade de início & Precoce & $\begin{array}{l}\text { Após os anticorpos } \\
\text { maternos serem } \\
\text { catabolizados (5-12 } \\
\text { meses) ou final da } \\
\text { infância }\end{array}$ & Precoce & Qualquer idade \\
\hline $\begin{array}{l}\text { Órgãos mais } \\
\text { afetados }\end{array}$ & $\begin{array}{l}\text { Crescimento } \\
\text { inadequado, diarreia } \\
\text { crônica, candidíase } \\
\text { persistente }\end{array}$ & $\begin{array}{l}\text { Infecções sino- } \\
\text { pulmonares, sintomas } \\
\text { gastrintestinais, má } \\
\text { absorção, artrites, } \\
\text { meningoencefalite }\end{array}$ & $\begin{array}{l}\text { Celulite, abscessos, } \\
\text { adenite, periodontite, } \\
\text { osteomielite }\end{array}$ & $\begin{array}{l}\text { Meningite, artrite, } \\
\text { septicemia, } \\
\text { infecções sino- } \\
\text { pulmonares }\end{array}$ \\
\hline $\begin{array}{l}\text { Características } \\
\text { especiais }\end{array}$ & $\begin{array}{l}\text { Doença enxerto versus } \\
\text { hospedeiro, causada } \\
\text { pelas células maternas } \\
\text { ou transfusão de } \\
\text { sangue não-irradiado, } \\
\text { BCGíte, tetania } \\
\text { hipocalcêmica }\end{array}$ & $\begin{array}{l}\text { Autoimunidade, linfoma, } \\
\text { timoma, paralisia pela } \\
\text { vacina oral contra } \\
\text { poliomielite }\end{array}$ & $\begin{array}{l}\text { Retardo na queda } \\
\text { do coto umbilical, } \\
\text { dificuldade de } \\
\text { cicatrização }\end{array}$ & $\begin{array}{l}\text { Vasculites, } \\
\text { lúpus sistêmico, } \\
\text { dermatomiosite, } \\
\text { glomerulonefrite, } \\
\text { angioedema }\end{array}$ \\
\hline
\end{tabular}

CMV: citomegalovirus; EBV: vírus Epstein-Bair

micobactérias e salmonelas ${ }^{(31)}$. Indivíduos portadores de deficiências dos componentes terminais do sistema complemento geralmente apresentam infecções por neissérias ${ }^{(27)}$. Deficiências fagocíticas cursam com infecções cutâneas, respiratórias, neurológicas e do sistema retículo-endotelial por estafilococos, bactérias gram negativas e fungos ${ }^{(25)}$.

Dessa forma, são aspectos essenciais para que o pediatra possa suspeitar de IDP:

- História e exame físico sugestivos;

- Infecções por patógenos específicos e/ou de baixa virulência;

- Concomitância de síndromes genéticas;

- História familiar positiva.

Com relação à história clínica, é importante questionar sobre a idade de início, a frequência e a gravidade das infecções, bem como quanto à presença de complicações pós-infecciosas, hospitalizações e reações vacinais graves. No que diz respeito ao exame físico, o pediatra deve estar atento à evolução pôndero-estatural e à presença de caracteres fenotípicos anormais (fácies, tipo de cabelo, presença de alterações cutâneas). Ausência de tonsilas palatinas (na ausência de cirurgia) ou de gânglios linfáticos, mesmo em vigência de infecções graves, é um forte indício de imunodeficiência combinada grave $e^{(25)}$.

No tocante à história familiar, deve-se questionar sobre consanguinidade entre os pais, antecedentes de infecções recorrentes, morte por infecções graves, neoplasias ou autoimunidade em outros membros da família e abortamento espontâneo de causa desconhecida na história materna.

O diagnóstico precoce das IDP é essencial para que medidas terapêuticas gerais e específicas sejam rapidamente instituídas, reduzindo os riscos de óbito e de complicações e hospitalizações. Com o intuito de facilitar o raciocínio clínico acerca dos pacientes que carecem de investigação laboratorial, a Fundação Jeffrey Modell, juntamente com a Cruz Vermelha Americana, publicaram, em 1999, os Dez Sinais de Alerta para IDP, adaptados ao nosso meio pelo Grupo Brasileiro de Imunodeficiência ${ }^{(32)}$ e divulgados pela Sociedade de Pediatria de São Paulo (Quadro 2). A presença de um ou mais desses 10 sinais de alerta torna obrigatória a investigação laboratorial para uma possível IDP. 
Quadro 2 - Os dez sinais de alerta para imunodeficiência primária na criança, adaptado da Fundação Jeffrey Modell(32)

1. Duas ou mais pneumonias no último ano
2. Quatro ou mais novas otites no último ano
3. Estomatites de repetição ou monilíase por mais de
dois meses
4. Abscessos de repetição ou ectima
5. Um episódio de infecção sistêmica grave (meningite,
osteoartrite, septicemia)
6. Infecções intestinais de repetição/diarreia crônica;
7. Asma grave, doença do colágeno ou doença
autoimune
8. Efeito adverso ao BCG e/ou infecção por
micobactéria
9. Fenótipo clínico sugestivo de síndrome associada à
imunodeficiência
10. História familiar de imunodeficiência

\section{Referências bibliográficas}

1. de Martino M, Ballotti S. The child with recurrent respiratory infections: normal or not? Pediatr Allergy Immunol 2007;18:13-8.

2. Conley ME, Stiehm ER. Immunodeficiency disorders: general considerations. In: Stiehm ER, editor. Immunologic disorders in infants and children. 4th ed. Philadelphia: Saunders; 1996. p. 201-49.

3. Nudelman V, Costa Carvalho BT, Ejzenberg B, Roxo Júnior P. A criança com infecção das vias aéreas superiores (rinites, otites, sinusites). In: Santos Vilela MM, Lotufo JP, editors. Alergia, imunologia e pneumologia. Série Atualizações Pediátricas. São Paulo: Atheneu; 2004. p. 37-45.

4. Berman S. Epidemiology of acute respiratory infections in children of developing countries. Rev Infec Dis 1991;6:S454-62.

5. Towns S, Wong M. Assessment of the child with recurrent respiratory infections. Aust Fam Physician 2000;29:741-5.

6. Juvonen R, Bloigu A, Peitso A, Silvennoinen-Kassinen S, Saikku P, Leinonen $M$. et al. Risk factors for acute respiratory tract illness in military conscripts. Respirology 2008;13:575-80.

7. Roxo Júnior $P$, Sorensen RU. Imunodeficiências predominantemente humorais. In: Roxo Júnior P, editor. Alergia e imunodeficiências em Pediatria: abordagem prática. Ribeirão Preto: Tecmedd; 2006. p. 237-70.

8. Stingone JA, Claudio L. Disparities in allergy testing and health outcomes among urban children with asthma. J Allergy Clin Immunol 2008;122:748-53.

9. Tolia V, Vandenplas Y. Systematic review: the extra-esophageal symptoms of gastro-esophageal reflux disease in children. Aliment Pharmacol Ther; Epub 2008 Oct 30

10. Filizzola VCC. Fatores de risco para amigdalite aguda de repetição em crianças. Rev Bras Alerg Imunopatol 1998;21:100-4.

11. Nafstad P, Hagen JA, Oie L, Magnus D, Jaakkola JJ. Day care centers and respiratory healthy. Pediatrics 1999; 103:753-8.

12. Genov IR, Roxo Júnior P. Otite média recorrente na infância. Medicina Ribeirão Preto 2001;34:297-300.

13. Hirata C. Fatores de risco em crianças com otite média recorrente. Rev Paul Pediatria 1999;17:15-9
Por outro lado, diversas condições também podem estar associadas às IDP, como quadros alérgicos graves, asma, neoplasias do sistema linfo-hematopoiético, autoimunidade, doença inflamatória crônica intestinal e endocrinopatias ${ }^{(25,33)}$.

Finalizando, as IDP representam um grande desafio em seu diagnóstico e tratamento. Seu prognóstico depende, sobretudo, do reconhecimento precoce dessas doenças. É de extrema importância que o pediatra esteja atento aos principais pontos de alerta para se pensar em IDP, de tal forma que os casos suspeitos sejam encaminhados para serviços de referência em Imunologia o mais precocemente possível. A adequada investigação diagnóstica e a adoção de medidas terapêuticas específicas são capazes de melhorar o prognóstico e a qualidade de vida desses pacientes.
14. Faden H, Duffy L, Boeve M. Otitis media: back to basics. Pediatr Infect Dis J 1998;17:1105-12.

15. Kiliç SS, Tezcan I, Sanal O, Metin A, Ersoy F. Transient hypogammaglobulinemia of infancy: clinical and immunologic features of 40 new cases. Pediatr Int 2000;42:647-50.

16. McGeady SJ. Transient hypogammaglobulinemia of infancy: need to reconsider name and definition. J Pediatr 1987;110:47-50.

17. Latiff AHA, Kerr, MA. The clinical significance of immunoglobulin A deficiency. Ann Clin Biochem 2007;44:131-9.

18. Hanson LA, Ahlstedt S, Andersson B, Carlsson B, Cole MF, Cruz JR et al Mucosal immunity. Ann N Y Acad Sci 1983;409:1-21.

19. Tagliabue A, Nencioni L, Villa L, Keren DF, Lowell GH, Boraschi D. Antibodydependent cell-mediated antibacterial activity of intestinal linfocytes with secretory IgA. Nature 1983;306:194-6.

20. Kyaw MH, Christie P, Clarke SC, Mooney JD, Ahmed S, Jones IG et al. Invasive pneumococcal disease in Scotland, 1999-2001: use of record linkage to explore associations between patients and disease in relation to future vaccination policy. Clin Infect Dis 2003;37:1283-91.

21. Miller E, Waight P, Efstratiou A, Brisson M, Johnson A, George R. Epidemiology of invasive and other pneumococcal disease in children in England and Wales 1996-1998. Acta Paediatr 2000;89:11-6.

22. Clarke SC. Control of pneumococcal disease in the United Kingdom - the start of a new era. J Med Microbiol 2006;55:975-80.

23. Black SB, Shinefield HR, Ling S, Hansen J, Fireman B, Spring D et al. Effectiveness of heptavalent pneumococcal conjugate vaccine in children younger than five years of age for prevention of pneumonia. Pediatr Infect Dis J 2002;21:810-5.

24. Fireman B, Black SB, Shinefield HR, Lee J, Lewis E, Ray P. Impact of the pneumococcal conjugate vaccine on otitis media. Pediatr Infect Dis $J$ 2003;22:10-6.

25. Morimoto Y, Routes JM. Immunodeficiency Overview. Prim Care Clin Office Pract 2008;35:159-73. 
26. Bruton OC. Agammaglobulinemia. Pediatrics 1952;9:722-8.

27. Bonilla FA, Bernstein IL, Khan DA, Chinen J, Frank MM, Kobrynski LJ et al. Practice parameter for the diagnosis and management of primary immunodeficiency. Ann Allergy Asthma Immunol 2005;94:S1-63.

28. Sáfadi MA, Roxo Júnior P. Imunizações em Pacientes Imunocomprometidos. In: Roxo Júnior P, editor. Alergia e Imunodeficiências em Pediatria-Abordagem Prática. Ribeirão Preto: Tecmedd; 2006. p. 374-401.

29. Woroniecka M, Ballow M. Office evaluation of children with recurrent infection. Pediatr Clin N Amer 2000;47:1211-24.
30. Cunningham-Rundles $C$, Bodian $C$. Common variable immunodeficiency: clinical and immunological features of 248 patients. Clin Immunol 1999;92:34-48.

31. Winkelstein JA, Marino MC, Ochs H, Fuleihan R, Scholl PR, Geha R et al The X-linked hyper-IgM syndrome: clinical and immunologic features of 79 patients. Medicine (Baltimore) 2003;82:373-84.

32. Costa Carvalho BT, Roxo Júnior $P$, Tavares FS. Como abordar a criança com infecção respiratória de repetição. Sociedade de Pediatria de São Paulo Atualização Científica 2008;24:343.

33. Chinen J, Anmuth D, Franklin AR, Shearer WT. Long-term follow-up of patients with primary immunodeficiencies. J Allergy Clin Immunol 2007;120:795-7. 